\title{
Higher Expectancy of Acupuncture Treatment is Associated with Significant Higher Ovulation, Less Bruising and Lower Dropout in Infertile Women with PCOS Receiving Acupuncture
}

\section{Yi Gong}

The First Affiliated College of Medicine, Beilun Branch of Zhejiang University

Jian Li

Affiliated Hospital of Guizhou Medical University

Hong-Li Ma

Department of Gynecology, the First Affifiliated Hospital, Heilongjiang University of Chinese Medicine, Harbin, China.

Hui He

Department of Gynecology, the First Affifiliated Hospital, Heilongjiang University of Chinese Medicine, Harbin, China.

Xiao-Ke Wu ( $\triangle$ xiaokewu2002@vip.sina.com )

Department of Gynecology, the First Affifiliated Hospital, Heilongjiang University of Chinese Medicine, Harbin, China.

\section{Research Article}

Keywords: polycystic ovary syndrome, acupuncture, expectancy, ovulation

Posted Date: December 16th, 2021

DOI: https://doi.org/10.21203/rs.3.rs-1134647/v1

License: (c) (1) This work is licensed under a Creative Commons Attribution 4.0 International License. Read Full License 


\section{Abstract}

Object: To study the expectancy of acupuncture treatment on fertility outcomes and adverse events in infertile women with polycystic ovary syndrome (PCOS) receiving acupuncture.

Method: This analysis including 1000 infertile women with PCOS. Women with available expectancy of acupuncture treatment were included in analysis, of whom were subdivided into low and high expectancy groups. The anthropometric and endocrinal parameters, fertility outcomes as well as adverse events and dropout were compared. The risk of low expectancy of acupuncture treatment on ovulation and correspondent $95 \%$ confidence interval was computed by multivariable logistic regression model. The Kaplan Meier survival curve was employed to plot the time to ovulation and Cox regression model was used to compare the difference of time to ovulation between groups, adjusting by interventions.

Results: 827 women were included in analysis, of whom 373 and 454 women were identified as low and high expectancy, respectively. Compared with low expectancy, women with high expectancy had higher BMI (24.56 vs $23.77 \mathrm{~kg} / \mathrm{m}^{2}, \mathrm{p}=0.03$ ), shorter time to attempt to conception (22.97 vs. 25.00 months, $\mathrm{p}=$ 0.03 ), earlier menarche (13.61vs.13.68 years, $p=0.03$ ) and lower estradiol $(256.68 \mathrm{vs} .284 .13 \mathrm{pmol} / \mathrm{L}, p=$ 0.03). There was not interaction between acupuncture treatment and expectancy of acupuncture treatment regarding ovulation $(p=0.07)$. High expectancy was associated with significant higher ovulation (adjusted odds ratio (OR) 2.33, 95\% Cl 1.31 to $4.16, \mathrm{p}=0.004$ ) and lower dropout (adjusted OR $0.13,95 \% \mathrm{Cl} 0.03$ to 0.57 ) in women receiving control acupuncture, even after adjustment by BMI, estradiol, and clomiphene, while it was associated with significant less bruising (adjusted OR $0.28,95 \% \mathrm{Cl}$ 0.13-0.61) and lower dropout (adjusted OR $0.42,95 \% \mathrm{Cl} 0.18-0.96$ ) in those receiving active acupuncture. High expectancy was associated with significant shorter time to ovulation (median 28 vs 49 days, $\mathrm{P}<$ 0.001 ) in women received control acupuncture, yet it was no longer significant after adjustment for clomiphene.

Conclusion: High expectancy of acupuncture treatment was associated with significant higher ovulation, less bruising and lower dropout, but not other fertility outcome in infertile women with PCOS receiving acupuncture.

Trial Registration:ClinicalTrial.gov number: NCT01573858 and chictr.org.cn number: ChiCTR-TRC12002081.

\section{Background}

Polycystic ovary syndrome (PCOS) is the most common endocrine disorder, affecting up to $20 \%$ reproductive-age women [1]. Women with PCOS are often characterized by ovarian dysfunction, androgen excess, and/or polycystic ovarian morphology (PCOM), leading to infertility,anxiety and depression symptoms [2]. In addition, PCOS is associated with increased risks of type 2 diabetes mellitus, cardiovascular diseases, or even endometrial carcinoma[3] (Deeks, Gibson-Helm et al. 2010), Teede, Deeks et al. (2010)(Deeks, Gibson-Helm et al. 2010, Teede, Deeks et al. 2010)[4]. 
Acupuncture, a key part of TCM, is getting attention in infertility treatment. It had been reported that electro-acupuncture (EA) can restore the menstrual cycle via increasing $\beta$-endorphin production in women with PCOS $[5,6][7,8]$. Apart from hormone biosynthesis, it was reported that EA also had effects on hypothalamic DNA methylation, the latter is considered to involving in the development of PCOS [9]. Though a meta-analysis suggested that acupuncture can not only promote the recovery of menstrual cycles but also reduce the circulating concentration of testosterone in women with PCOS, the ovulation and live birth are not significantly improved [10]. Up to now, it is still lack of proven evidence to support the effectiveness of acupuncture on infertility [11].

In general, it is considered that both nonspecific and specific effects are existing during acupuncture [1214]. Acupuncture exerts effectiveness by stimulating acupoints that is called specific efficacy. For nonspecific effects, it is mainly consist of 4 aspects: a) the patient's perception of the acupuncturist; $b$ ) the patient's knowledge, attitudes, and behaviors; c) the patient-acupuncturist relationship; and d) the trial environment [15], of which the patient's expectancies of acupuncture is important. Previously, it was reported that expectancies of acupuncture improved pain independently of the acupuncture [16, 17]. Whereas a study suggested that expectancies of acupuncture was not significantly associated with outcome, yet the acupuncture is noninsertive stimulation of acupuncture points, and the expectancies of acupuncture were provided before randomization [18].

Therefore, we hypothesized that high expectation is significantly associated with higher ovulation, shorten time to ovulation, and less adverse events. We aimed to study the associations between the expectation of acupuncture and infertility outcomes, time to ovulation and adverse events in infertile women with PCOS who underwent ovulation induction by active or control acupuncture with/without clomiphene.

\section{Methods}

\section{Participants}

This study was a secondary analysis of Polycystic Ovary Syndrome Acupuncture plus clomiphene trial (PCOSAct) whch was a large, multi-center, randomized controlled trial. The trial was registered on both ClinicalTrials.gov (NCT01573858) and chictr.org.cn (ChiCTR-TRC-12002081). PCOSAct included 1000 participants who were diagnosed with PCOS according to the modified Rotterdam criteria, of whom received active or sham acupuncture with or without clomiphene up to four months. All sites have passed the ethical review, and the study protocol and the main results had been published [19, 20].

\section{Demographic characteristics}

Baseline demographic data included age, body mass index (BMI), mean menstrual interval, time attempting to conceive, Self-Rating Anxiety Scale, Self-Rating Depression Scale. Hirsutism was assessed via the modified Ferriman-Gallwey hirsutism score. Acne was measured by definitions and a standard acne lesion assessment diagram. 


\section{Biochemical measurement}

Biochemical measurements including luteinizing hormone (LH), follicle stimulating hormone (FSH), total testosterone (TT), free testosterone (FT), sex hormone binding globulin (SHBG), estradiol (E2), progesterone $(\mathrm{P})$, anti-mullerian hormone $(\mathrm{AMH})$, fasting glucose and insulin. Free androgen index (FAI) and testosterone/estradiol ratio (T/E2) were calculated.

\section{Interventions}

Acupuncture protocols was made according to the CONSORT [21] and STRICTA [22]. For active acupuncture, two sets of acupoints were alternately used every other section, the acupoints was described detailly in Supplement table 1. [19]The needles were inserted with a depth of 15-35 mm and then stimulated with current of $2 \mathrm{~Hz}, 0.3 \mathrm{~ms}$ pulse length. Manipulation was stimulated every 10 minutes (deqi) for a total of 4 times. In control acupuncture, the acupoints selected which have no effect on the ovulation function in women with PCOS, the needles will be inserted superficially with a depth of $<5 \mathrm{~mm}$ in each arm. To mimic active electroacupuncture, the needle was connected to the electrode, while the stimulation intensity was 0 (no current) and the process of manual stimulation was also absent.

Clomiphene citrate (CC) administration started at $50 \mathrm{mg}$ and the dosage was gradually increased to no more than $150 \mathrm{mg}$ in women who did not respond; the length of treatment was four cycles.

\section{Expectation scale of acupuncture}

Participants completed the expectation scale of acupuncture after receiving three sections of acupuncture. The scale was described in detail in supplement table 2. The answer was divided into fivegrade category, including 1) Not at all, 2) Not very, 3) Neither, 4) Quite, 5) Very confident. One to five points were coded from "Not at all" to "Very confident", the higher score the higher expectation. The high expectation was defined as each item with equal or more than 4 points, otherwise was low expectation.

\section{Fertility outcomes}

Ovulation was defined as serum progesterone level above $3 \mathrm{ng} / \mathrm{mL}$. Conception was defined as any positive serum hCG test. Pregnancy was defined as intrauterine pregnancy and fetal heart can be seen under ultrasound. Live birth was defined as delivery of a viable infant. Pregnancy loss was defined as all intrauterine pregnancies around 20 weeks, including miscarriage, fetal dysplasia, and stillbirth.

\section{Adverse events and drop out}

Adverse events of acupuncture mainly include bruising and local skin itching, bleeding, pian. Drop out was defined as treatment break off for more than 4 weeks.

\section{Statistical analysis}


A total of 827 women were included in the analysis. All statistical analyses were conducted by SAS version 9.4 Software (SAS Institute Inc). Data were described as mean (SD) for continuous variables and frequencies (percent) for categorical variables The baseline characteristics between the high- and lowexpectation groups were compared by Mann-whitney $\mathrm{U}$ test. The comparison of reproductive outcomes, adverse events and drop-out rates between groups were analyzed by Chi-square test or Fisher's exact test and logistic regression model was used to calculate the odds ratio (OR) and 95 percentage of confident interval. The Kaplan Meier survival curve was employed to calculate time to ovulation between high- and low-expectation groups.

\section{Results}

827 patients were included in the analysis, 373 and 454 women were low- and high expectation, respectively. The points of expectation scale in total ranged from 4 to 20 , the frequencies of participants for each point were shown in detail in Figure 1.

Compared with low expectancy, women with high expectancy had higher BMI (24.56 vs. $23.77 \mathrm{~kg} / \mathrm{m}^{2}, \mathrm{p}=$ 0.03 ), shorter time to attempt to conception (22.97 vs. 25.00 months, $p=0.03$ ), earlier menarche (13.61 vs.13.68 years $p=0.03)$, and lower estradiol ( 256.68 vs. $284.13 \mathrm{pmol} / \mathrm{L}, \mathrm{p}=0.03)$ (Table1).

There was not interaction between acupuncture and expectation of acupuncture treatment regarding ovulation ( $p=0.07)$. The high expectancy was associated with significant higher ovulation (adjusted odds ratio (OR) $2.33,95 \% \mathrm{Cl} 1.31$ to $4.16, \mathrm{p}=0.004$ ) in women receiving control acupuncture rather those receiving active acupuncture, even after adjustment by BMI, estradiol, and clomiphene. While high expectancy was associated with significant less bruising (adjusted OR $0.28,95 \% \mathrm{Cl} 0.13-0.61, \mathrm{p}=0.001$ ) in women receiving active acupuncture. Moreover, higher expectancy was associated with significant lower dropout in both active (adjusted OR $0.42,95 \% \mathrm{Cl} 0.18-0.96$ ) and control acupuncture (adjusted OR 0.13, 95\% Cl 0.03-0.57) groups (Table2),

The high expectancy was associated with significant shorter time to ovulation (median 28 vs 49 days, $\mathrm{P}<0.001$ ) in women with control acupuncture (Figure2), while it was no longer significant after adjustment for clomiphene.

\section{Discussion}

In this study, we found that high expectancy of acupuncture treatment was associated with significant higher ovulation and lower dropout in infertile women with PCOS receiving control acupuncture, while it was associated with significant less bruising and lower dropout in women receiving active acupuncture. After adjusting clomiphene, the time to ovulation did not significantly differ between high- and lowexpectancy, regardless of active or control acupuncture. There was no interaction between acupuncture treatment and its expectation regarding ovulation. 
In this study, the strength was that we accessed the expectancy of acupuncture treatment on infertility outcomes in infertile women with PCOS receiving acupuncture, based on a large sample, well-designed, randomized controlled trial. Moreover, the acupuncturist in this study was trained and qualified prior to performing the acupuncture which can minimize the bias of expectancy assessed by participants. There were a few limitations in our study. Firstly, the expectation scale was merely developed by acupuncture experts, it may not precisely describe the expectancy of acupuncture treatment, without participant involvement. Secondly, the scale was finished as participants received 3 sections of acupuncture, this may impair the expectancy in women who received control acupuncture or experienced adverse events.

Previously, there was not consistent evidence regarding the association of expectancies of acupuncture with clinical outcome such as chronic pain. In Linde's study [16], it indicated that expectancy of acupuncture was independently associated with pain relief, and its effects was stronger in patients receiving active acupuncture than those with the control. However, another study shown that there was no association between expectancy of acupuncture and pain relief .[18].[23]Therefore, the discrepancies may attribute to the time of expectancy assessment and type of control acupuncture, the latter can be designed as a sham acupuncture (superficial insertion bypass acupoints) or non-penetration with blunt needle. Previously, it had been demonstrated that high expectancy produced analgesia effects liking placebo, involving in the emotional circuit modulation [24, 25]. Here, we found that there was associations between high expectancy and ovulation in women receiving control acupuncture (superficial insertion), it implied that the effects of high expectancy on ovulation was probably independent of acupuncture treatment, involving in emotional modulation such as anxiety or depression.

Interesting, the differences of ovulation between low and high expectancy was missing in those women who received active acupuncture. It had reported that acupuncture can restore menstrual cycle via increasing $\beta$-endorphin production and.improve polycystic ovary morphology [5] [10]. In addition, acupuncture can improve symptoms of anxiety/depression and quality of life in women with PCOS, with reducing circulating concentration of serotonin (5-HT) [3] [26], eventually resulting in improvement of ovulation disorder. Therefore, we speculated that the harm effects of low expectancy on ovulation was compensated by active acupuncture, consequently, the effects of expectancy of acupuncture on ovulation was no longer significant.

In general, adverse events for patients receiving active acupuncture included bruising, bleeding and itching and pain, occurring serious adverse events was rare. Here, we found that the bruising (adjusted OR $0.28,95 \% \mathrm{Cl}$ 0.13-0.61) was significant lower in women with high expectancy than their counterpart, namely, women with low expectancy were more likely to experience adverse events, this implied that low expectancy was associated with not only lower ovulation but also more frequency of adverse events. Also, women with low expectancy had high dropout rates, regardless of active acupuncture. Consistent with our result, it had been reported that dropout was higher in patients with lower expectation as compare with those with high expectancy [27], even if the patient receives a "placebo" treatment [28]. Though there are still lack of proven evidence regarding live birth, acupuncture is frequently administered to infertile women in recent years, providing additional clinical benefits such as anxiety. In this condition, 
to measure the expectation of treatment prior to acupuncture provide a clue for the physicians to justify whether some approaches to enhance the expectancy of acupuncture should be provided.

In summary, higher expectancy of acupuncture treatment is associated with higher ovulation and less bruising and dropout in infertile women with PCOS receiving acupuncture, but not other fertility outcomes and time to ovulation.

\section{Declarations}

\section{Ethics approval and consent to participate}

The PCOSAct trial was approved by the Heilongjiang University of Chinese Medicine First Affifiliated Hospital Ethics Committee (2010HZYLL-010). Approval was granted on 15 December 2011.

\section{Consent for publication}

Not applicable.

\section{Availability of data and materials}

The datasets are not publicly available due privacy or ethical restrictions, but are available from the corresponding author on reasonable request.

\section{Competing interests}

All authors have no conflict of interests.

\section{Funding}

National Public Welfare Projects for Chinese Medicine (201507001).

\section{Authors' contributions}

YG, JL, and XKW: Contributions to conception and design. PCOSact Study group: Acquisition of the data. YG and JL : Analysis and interpretation of data. YG,JL and $\mathrm{HH}$ : Drafting the article. HLM and XKW : Revising the article critically for important intellectual content. All authors critically revised the manuscript and approved the final version.

\section{Acknowledgements}

We are thankful to all the people who provided data for this study and the PCOSact team.

\section{References}


1. Escobar-Morreale, H.F., Polycystic ovary syndrome: definition, aetiology, diagnosis and treatment. Nat Rev Endocrinol, 2018. 14(5): p. 270-284.

2. Karjula, S., et al., Psychological Distress Is More Prevalent in Fertile Age and Premenopausal Women With PCOS Symptoms: 15-Year Follow-Up. J Clin Endocrinol Metab, 2017. 102(6): p. 1861-1869.

3. Deeks, A.A., M.E. Gibson-Helm, and H.J. Teede, Anxiety and depression in polycystic ovary syndrome: a comprehensive investigation. Fertil Steril, 2010. 93(7): p. 2421-3.

4. Teede, H., A. Deeks, and L. Moran, Polycystic ovary syndrome: a complex condition with psychological, reproductive and metabolic manifestations that impacts on health across the lifespan. BMC Med, 2010. 8: p. 41.

5. Chen, H. and C.E.D. Lim, The efficacy of using acupuncture in managing polycystic ovarian syndrome. Curr Opin Obstet Gynecol, 2019. 31(6): p. 428-432.

6. Li, D., et al., Effect of Acupuncture on Ovary Morphology and Function in DHEA-Induced Polycystic Ovary Syndrome Model Rats. Chin J Integr Med, 2021. 27(3): p. 220-224.

7. Leonhardt, H., et al., Serum anti-Mullerian hormone and ovarian morphology assessed by magnetic resonance imaging in response to acupuncture and exercise in women with polycystic ovary syndrome: secondary analyses of a randomized controlled trial. Acta Obstet Gynecol Scand, 2015. 94(3): p. 279-87.

8. Ee, C., et al., Feasibility and acceptability of a proposed trial of acupuncture as an adjunct to lifestyle interventions for weight loss in Polycystic Ovary Syndrome: a qualitative study. BMC Complement Altern Med, 2018. 18(1): p. 298.

9. Cui, P., et al., Hypothalamic DNA methylation in rats with dihydrotestosterone-induced polycystic ovary syndrome: effects of low-frequency electro-acupuncture. Exp Physiol, 2018. 103(12): p. 16181632.

10. Wu, J., D. Chen, and N. Liu, Effectiveness of acupuncture in polycystic ovary syndrome: A systematic review and meta-analysis of randomized controlled trials. Medicine (Baltimore), 2020. 99(22): p. e20441.

11. Lim, C.E.D., et al., Acupuncture for polycystic ovarian syndrome. Cochrane Database Syst Rev, 2019. 7: p. CD007689.

12. MacPherson, H., L. Thorpe, and K. Thomas, Beyond needling-therapeutic processes in acupuncture care: a qualitative study nested within a low-back pain trial. J Altern Complement Med, 2006. 12(9): p. 873-80.

13. Paterson, C. and P. Dieppe, Characteristic and incidental (placebo) effects in complex interventions such as acupuncture. BMJ, 2005. 330(7501): p. 1202-5.

14. White, A.R., et al., Clinical trials of acupuncture: consensus recommendations for optimal treatment, sham controls and blinding. Complement Ther Med, 2001. 9(4): p. 237-45.

15. Ho, R.S., et al., Non-specific effects of acupuncture and sham acupuncture in clinical trials from the patient's perspective: a systematic review of qualitative evidence. Acupunct Med, 2021. 39(1): p. 319. 
16. Linde, K., et al., The impact of patient expectations on outcomes in four randomized controlled trials of acupuncture in patients with chronic pain. Pain, 2007. 128(3): p. 264-271.

17. Kalauokalani, D., et al., Lessons from a trial of acupuncture and massage for low back pain: patient expectations and treatment effects. Spine (Phila Pa 1976), 2001. 26(13): p. 1418-24.

18. Sherman, K.J., et al., Treatment expectations and preferences as predictors of outcome of acupuncture for chronic back pain. Spine (Phila Pa 1976), 2010. 35(15): p. 1471-7.

19. Kuang, H., et al., Acupuncture and clomiphene citrate for live birth in polycystic ovary syndrome: study design of a randomized controlled trial. Evid Based Complement Alternat Med, 2013. 2013: p. 527303.

20. Wu, X.K., et al., Effect of Acupuncture and Clomiphene in Chinese Women With Polycystic Ovary Syndrome: A Randomized Clinical Trial. JAMA, 2017. 317(24): p. 2502-2514.

21. Moher, D., et al., The CONSORT Statement: revised recommendations for improving the quality of reports of parallel-group randomized trials 2001. Explore (NY), 2005. 1(1): p. 40-5.

22. MacPherson, $\mathrm{H}$., et al., Revised STandards for Reporting Interventions in Clinical Trials of Acupuncture (STRICTA): extending the CONSORT statement. Acupunct Med, 2010. 28(2): p. 83-93.

23. Bertisch, S.M., et al., The impact of psychological factors on placebo responses in a randomized controlled trial comparing sham device to dummy pill. J Eval Clin Pract, 2009. 15(1): p. 14-9.

24. Kong, J., et al., Expectancy and treatment interactions: a dissociation between acupuncture analgesia and expectancy evoked placebo analgesia. Neuroimage, 2009. 45(3): p. 940-9.

25. Kong, J., et al., An fMRI study on the interaction and dissociation between expectation of pain relief and acupuncture treatment. Neuroimage, 2009. 47(3): p. 1066-76.

26. Wang, Z., et al., Effects of electroacupuncture on anxiety and depression in unmarried patients with polycystic ovarian syndrome: secondary analysis of a pilot randomised controlled trial. Acupunct Med, 2019. 37(1): p. 40-46.

27. Reis B F, Brown L Gप2006ロ., Preventing therapy dropout in the real world: The clinical utility of videotape preparation and client estimate of treatment duration.. Professional Psychology Research \& Practice, 2006, 37(3):311-316.

28. Simpson, S. H., D. T. Eurich, S. R. Majumdar, R. S. Padwal, R. T. Tsuyuki, J. Varney and J. A. Johnson (2006). A meta-analysis of the association between adherence to drug therapy and mortality. BMJ 333(7557): 15.

\section{Tables}


Table 1

Comparison of baseline characteristics of between high- and low-expectations

\begin{tabular}{|c|c|c|c|}
\hline \multirow[t]{2}{*}{ Characteristic } & \multicolumn{2}{|c|}{ expectations and credibility } & \multirow[t]{2}{*}{$P$ value } \\
\hline & Low (n=373) & High $(n=454)$ & \\
\hline Age (years) & $27.86 \pm 3.37$ & $28.02 \pm 3.31$ & 0.58 \\
\hline $\mathrm{BMI}\left(\mathrm{kg} / \mathrm{m}^{2}\right)$ & $23.77 \pm 4.14$ & $24.56 \pm 4.28$ & $0.02 *$ \\
\hline Menarche (years) & $13.85 \pm 1.50$ & $13.61 \pm 1.42$ & $0.03 *$ \\
\hline Mean menstrual interval per year & $69.53 \pm 40.64$ & $70.21 \pm 46.44$ & 0.82 \\
\hline Mean menstrual cycle per year (days) & $6.20 \pm 2.22$ & $6.14 \pm 1.94$ & 0.79 \\
\hline Time attempting to conceive (month) & $25.00 \pm 17.36$ & $22.97 \pm 17.88$ & $0.03 *$ \\
\hline Self-Rating Anxiety Scale & $31.80 \pm 6.37$ & $32.58 \pm 6.72$ & 0.10 \\
\hline Self-Rating Depression Scale & $36.68 \pm 8.44$ & $37.29 \pm 8.86$ & 0.25 \\
\hline Ferriman-Gallwey hirsutism scoreł & $3.01 \pm 2.88$ & $2.95 \pm 2.65$ & 0.89 \\
\hline Acne score & $0.38 \pm 0.69$ & $0.48 \pm 0.80$ & 0.06 \\
\hline Luteinizing hormone (mlU/ml) & $10.46 \pm 5.75$ & $10.56 \pm 5.91$ & 0.91 \\
\hline Follicle stimulating hormone (mIU/ml) & $6.10 \pm 1.68$ & $6.15 \pm 1.65$ & 0.71 \\
\hline Estradiol (pmol/L) & $284.13 \pm 378.40$ & $256.68 \pm 240.40$ & $0.03 *$ \\
\hline Progesterone (ng/ml) & $2.66 \pm 5.38$ & $2.24 \pm 3.44$ & 0.98 \\
\hline Testosterone $(\mathrm{nmol} / \mathrm{l})$ & $1.68 \pm 0.67$ & $1.65 \pm 0.64$ & 0.48 \\
\hline Free testosterone $(\mathrm{pmol} / \mathrm{l})$ & $2.21 \pm 0.82$ & $2.33 \pm 0.86$ & 0.13 \\
\hline $\mathrm{AMH}(\mathrm{ng} / \mathrm{ml})$ & $12.18 \pm 6.68$ & $11.85 \pm 6.20$ & 0.70 \\
\hline Sex hormone-binding globulin $(\mu \mathrm{g} / \mathrm{mL})$ & $43.79 \pm 32.81$ & $41.51 \pm 28.25$ & 0.57 \\
\hline Free androgen index & $5.92 \pm 4.72$ & $5.79 \pm 4.27$ & 0.78 \\
\hline Fasting glucose (mmol/L) & $5.06 \pm 1.00$ & $5.03 \pm 1.02$ & 0.60 \\
\hline Fasting insulin (pmol/L) & $94.06 \pm 81.71$ & $101.36 \pm 92.26$ & 0.38 \\
\hline \multicolumn{4}{|c|}{ Mean (SD) are presented median \pm Standard Deviation. } \\
\hline Free androgen index $=(\mathrm{TT} / \mathrm{SHBG}) \times 100$ & & & \\
\hline
\end{tabular}


Table 2

The relationship between expectation on acupuncture treatment and ovulation in acupuncture(control/active)

\begin{tabular}{|c|c|c|c|c|c|c|c|c|}
\hline \multirow{3}{*}{$\begin{array}{l}\text { Fertility } \\
\text { outcomes } \\
\text { and } \\
\text { adverse } \\
\text { events } \\
\text { events }\end{array}$} & \multicolumn{8}{|c|}{ expectation } \\
\hline & \multicolumn{4}{|c|}{ Active acupuncture } & \multicolumn{4}{|c|}{ Control acupuncture } \\
\hline & $\begin{array}{l}\text { Low } \\
(n=168)\end{array}$ & $\begin{array}{l}\text { High } \\
(n=250)\end{array}$ & $\begin{array}{l}\text { OR } \\
\text { and } \\
95 \% \mathrm{Cl}\end{array}$ & $\begin{array}{l}\text { Adjusted } \\
\text { OR }\end{array}$ & $\begin{array}{l}\text { Low } \\
(n=205)\end{array}$ & $\begin{array}{l}\text { High } \\
(n=204)\end{array}$ & $\begin{array}{l}\text { OR } \\
\text { and } \\
95 \% \mathrm{Cl}\end{array}$ & $\begin{array}{l}\text { Adjusted } \\
\text { OR }\end{array}$ \\
\hline \multicolumn{9}{|l|}{$\begin{array}{l}\text { fertility } \\
\text { outcomes* }\end{array}$} \\
\hline Ovulation & $\begin{array}{l}129 \\
(76.7 \%)\end{array}$ & $\begin{array}{l}204 \\
(81.6 \%)\end{array}$ & $\begin{array}{l}1.34 \\
(0.83- \\
2.17)\end{array}$ & $\begin{array}{l}1.54 \\
(0.88- \\
2.69)\end{array}$ & $\begin{array}{l}147 \\
\text { (71.7\%) }\end{array}$ & $\begin{array}{l}177 \\
(86.7 \%)\end{array}$ & $\begin{array}{l}2.59 \\
(1.56- \\
4.29)\end{array}$ & $\begin{array}{l}2.33 \\
(1.31- \\
4.16)\end{array}$ \\
\hline Conception & $\begin{array}{l}51 \\
(30.3 \%)\end{array}$ & $\begin{array}{l}85 \\
(34 \%)\end{array}$ & $\begin{array}{l}1.20 \\
(0.79- \\
1.82)\end{array}$ & $\begin{array}{l}1.23 \\
(0.76- \\
1.98)\end{array}$ & $\begin{array}{l}58 \\
(28.2 \%)\end{array}$ & $\begin{array}{l}75 \\
(36.7 \%)\end{array}$ & $\begin{array}{l}1.47 \\
(0.97- \\
2.24)\end{array}$ & $\begin{array}{l}1.43 \\
(0.90- \\
2.29)\end{array}$ \\
\hline $\begin{array}{l}\text { Clinical } \\
\text { Pregnancy }\end{array}$ & $\begin{array}{l}33 \\
(19.6 \%)\end{array}$ & $\begin{array}{l}59 \\
(23.5 \%)\end{array}$ & $\begin{array}{l}1.27 \\
(0.78- \\
2.04)\end{array}$ & $\begin{array}{l}1.46 \\
(0.84- \\
2.53)\end{array}$ & $\begin{array}{l}38 \\
(18.5 \%)\end{array}$ & $\begin{array}{l}53 \\
(25.9 \%)\end{array}$ & $\begin{array}{l}1.54 \\
(0.96- \\
2.47)\end{array}$ & $\begin{array}{l}1.28 \\
(0.76- \\
2.15)\end{array}$ \\
\hline Live birth & $\begin{array}{l}29 \\
(17.2 \%)\end{array}$ & $\begin{array}{l}57 \\
(22.8 \%)\end{array}$ & $\begin{array}{l}1.79 \\
(1.08- \\
2.95)\end{array}$ & $\begin{array}{l}1.75 \\
(0.98- \\
3.12)\end{array}$ & $\begin{array}{l}37 \\
(18 \%)\end{array}$ & $\begin{array}{l}49 \\
(24 \%)\end{array}$ & $\begin{array}{l}1.44 \\
(0.90- \\
2.32)\end{array}$ & $\begin{array}{l}1.24 \\
(0.73- \\
2.10)\end{array}$ \\
\hline \multicolumn{9}{|l|}{$\begin{array}{l}\text { Adverse } \\
\text { events }\end{array}$} \\
\hline Bruising & $\begin{array}{l}23 \\
(13.6 \%)\end{array}$ & $\begin{array}{l}13 \\
(5.2 \%)\end{array}$ & $\begin{array}{l}0.35 \\
(0.17- \\
0.70)\end{array}$ & $\begin{array}{l}0.28 \\
(0.13- \\
0.61)\end{array}$ & $\begin{array}{l}7 \\
(3.4 \%)\end{array}$ & $\begin{array}{l}2 \\
(0.9 \%)\end{array}$ & $\begin{array}{l}0.28 \\
(0.06- \\
1.37)\end{array}$ & $\begin{array}{l}0.45(0.08- \\
2.64)\end{array}$ \\
\hline $\begin{array}{l}\text { Local skin } \\
\text { itching, } \\
\text { bleeding, } \\
\text { pian }\end{array}$ & $\begin{array}{l}2 \\
(1.1 \%)\end{array}$ & $10(4 \%)$ & $\begin{array}{l}3.46 \\
(0.75- \\
15.99)\end{array}$ & $\begin{array}{l}3.81 \\
(0.74- \\
19.59)\end{array}$ & $\begin{array}{l}7 \\
(3.4 \%)\end{array}$ & $\begin{array}{l}4 \\
(1.9 \%)\end{array}$ & $\begin{array}{l}0.57 \\
(0.16- \\
1.96)\end{array}$ & $\begin{array}{l}0.32 \\
(0.08- \\
1.34)\end{array}$ \\
\hline Drop out* & $\begin{array}{l}19 \\
(11.3 \%)\end{array}$ & $\begin{array}{l}11 \\
(4.3 \%)\end{array}$ & $\begin{array}{l}0.36 \\
(0.17- \\
0.78)\end{array}$ & $\begin{array}{l}0.42 \\
(0.18- \\
0.96)\end{array}$ & $\begin{array}{l}16 \\
(7.8 \%)\end{array}$ & $\begin{array}{l}4 \\
(1.9 \%)\end{array}$ & $\begin{array}{l}0.24 \\
(0.08- \\
0.72)\end{array}$ & $\begin{array}{l}0.13 \\
(0.03- \\
0.57)\end{array}$ \\
\hline
\end{tabular}

\section{Figures}




\section{Expectation and credibility of acupuncture treatment}

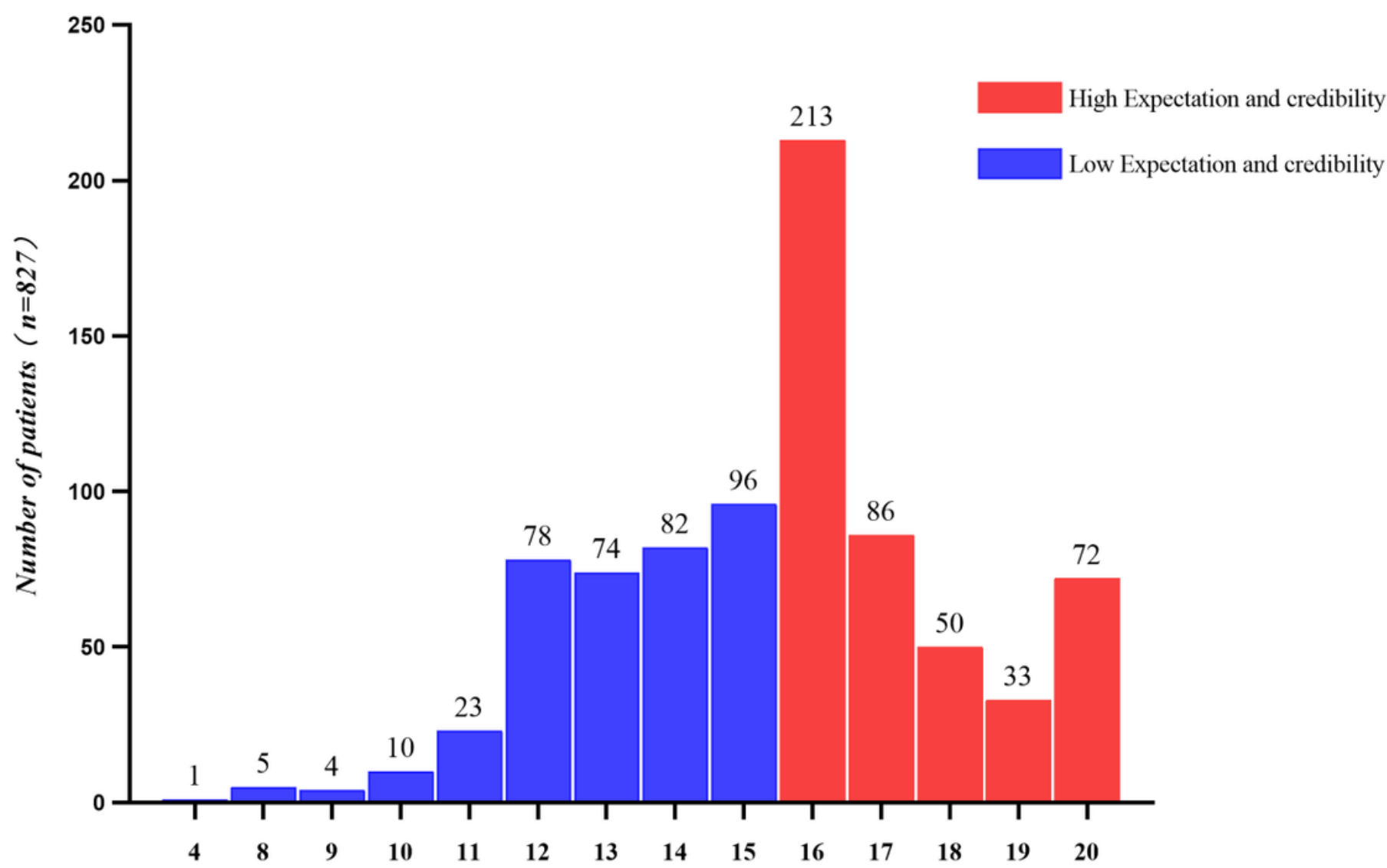

Total score

Figure 1

Sample frequency of expectations and credibility scores
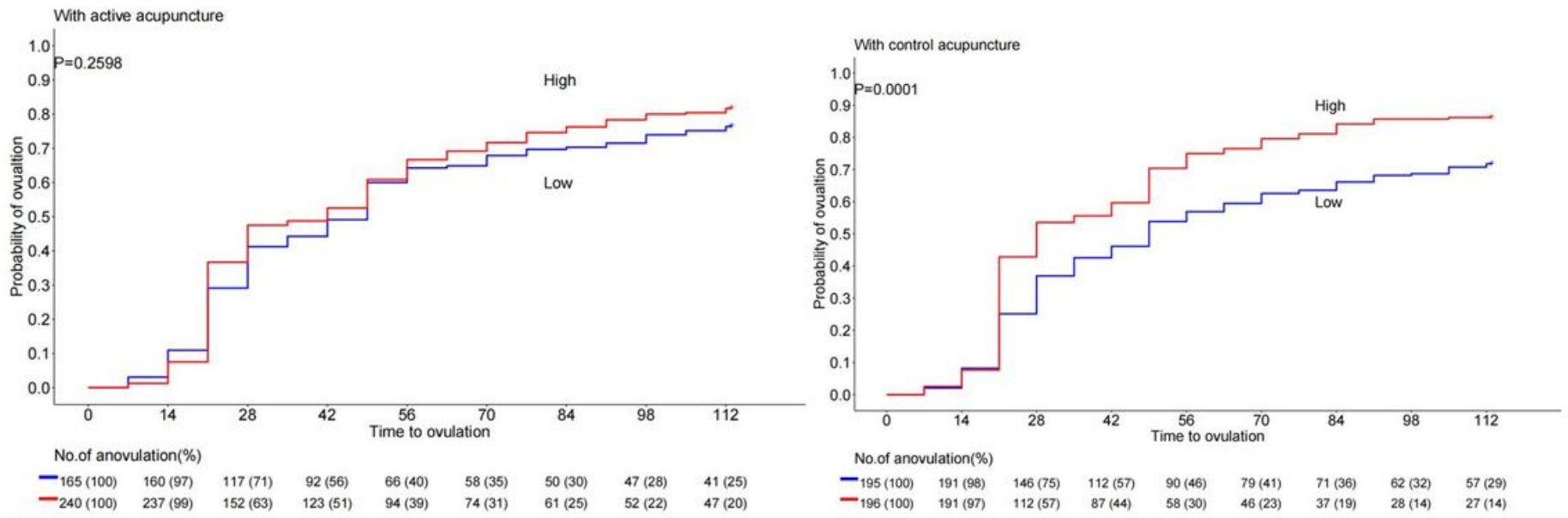
Figure 2

$\mathrm{KM}$ curves of different expectation levels and ovulation time.

\section{Supplementary Files}

This is a list of supplementary files associated with this preprint. Click to download.

- SupplementTable.doc 